\title{
Chemical abundances in six nearby star-forming regions ${ }^{\star}$
}

\section{Implications for galactic evolution and planet searches around very young stars}

\author{
N. C. Santos ${ }^{1}$, C. Melo ${ }^{2}$, D. J. James ${ }^{3}$, J. F. Gameiro ${ }^{1,4}$, J. Bouvier ${ }^{5}$, and J. I. Gomes ${ }^{6}$ \\ ${ }^{1}$ Centro de Astrofísica, Universidade do Porto, Rua das Estrelas, 4150-762 Porto, Portugal \\ e-mail: nuno@astro.up.pt \\ 2 European Southern Observatory (ESO), Casilla 19001, Santiago 19, Chile \\ 3 Department of Physics \& Astronomy, Box 1807 Station B, Vanderbilt University, Nashville, TN 37235, USA \\ 4 Departamento de Matemática Aplicada, Faculdade de Ciências da Universidade do Porto, Portugal \\ 5 Laboratoire d'Astrophysique, Observatoire de Grenoble, BP 53, 38041 Grenoble, France \\ ${ }^{6}$ Centro de Astrofísica da Universidade de Lisboa, Tapada da Ajuda, 1349-018 Lisboa, Portugal \\ Received 16 November 2007 / Accepted 10 January 2008
}

\section{ABSTRACT}

\begin{abstract}
In this paper we present a study of chemical abundances in six star-forming regions. Stellar parameters and metallicities are derived using high-resolution, high $\mathrm{S} / \mathrm{N}$ spectra of weak-line T-Tauri stars in each region. The results show that nearby star-forming regions have a very small abundance dispersion (only $0.033 \mathrm{dex}$ in $[\mathrm{Fe} / \mathrm{H}]$ ). The average metallicity found is slightly below that of the Sun, although compatible with solar once the errors are taken into account. The derived abundances for Si and Ni show that the observed stars have the abundances typical of Galactic thin disk stars of the same metallicity. The impact of these observations is briefly discussed in the context of the Galactic chemical evolution, local inter-stellar medium abundances, and in the origin of metal-rich stars in the solar neighbourhood (namely, stars more likely to harbour planets). The implication for future planet-search programmes around very young, nearby stars is also discussed.
\end{abstract}

Key words. planetary systems: formation - ISM: abundances - stars: abundances - Galaxy: abundances - solar neighborhood stars: formation

\section{Introduction}

As of November 2007, about 250 extra-solar planets have been discovered orbiting solar-type stars ${ }^{1}$, most of which were detected thanks to the development of high precision radialvelocity instruments (for a review see Udry \& Santos 2007). Complementary high accuracy spectroscopic studies have shown that stars hosting giant planets are particularly metal-rich when compared with single field stars (e.g. Gonzalez 1997; Gonzalez et al. 2001; Santos et al. 2001, 2004, 2005; Fischer \& Valenti 2005). This observation is helping to better understand the processes of planet formation and evolution.

The high metal content of some of the planet-hosts has raised a number of interesting questions regarding their origin. It has been proposed that the high metallicities could be the result of the in-fall of planetary (metal-rich) material into the stellar outer convective envelope (e.g. Gonzalez 1998; Murray et al. 1998), although current results do not support this hypothesis (Santos et al. 2004, 2005; Fischer \& Valenti 2005). The excess metallicity observed likely reflects the higher (average) metal abundances of the clouds of gas and dust that originated the star and

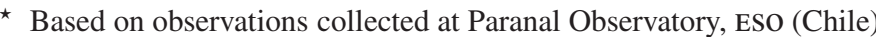
with the UVES and FLAMES/UVES spectrographs at the VLT-Kueyen telescope (run IDs 075.C-0272(A) and 076.C-0524(A), respectively), with the FEROS spectrograph at the ESO/MPI 2.2-m telescope (program ID 070.C-0507(A)), as well as with the SOPHIE spectrograph at the OHP observatory, France.

${ }^{1}$ For a continuously updated list see tables at

http://www. exoplanets. eu or http://www. exoplanet.eu
}

planet systems. Such conclusions are also supported by the most recent planet formation models (Ida \& Lin 2004; Benz et al. 2006).

Because the large majority of planet hosting stars are solarneighbourhood thin-disk objects (e.g. Ecuvillon et al. 2007), one would expect nearby T-Tauri stars and star-forming regions [SFRs] that are metal-rich to exist. Their existence is expected if a well-defined age-metallicity relation exists in the solarneighbourhood (Pont \& Eyer 2004; Haywood 2006). Previous studies (Padgett 1996; James et al. 2006), however, have not succeeded in finding evidence of high metallicity, T-Tauri stars in nearby SFRs. Whether this is due to the absence of such regions, or to the relatively small number of stars and SFRs observed was not clear.

Considering the strong metallicity-giant planet connection, the detection of nearby, metal-rich SFRs would provide preferential targets for future planet searches around young T-Tauri stars. The detection of planets orbiting T-Tauri stars would provide observational constraints of paramount importance for the study of planet formation scenarios, since it gives us some hint about the timescales for planetary formation.

In this paper, we continue the metallicity programme started in James et al. (2006), by increasing the number of observed young T-Tauri stars in each SFR, and by adding abundance determinations for objects that have not yet been studied. In all, we present accurate stellar parameters and chemical abundances for 19 weak line T-Tauri stars [WTTSs] in six nearby SFRs, namely Chamaeleon, Corona Australis, Lupus, Rho-Ophiuchus, Taurus 
and the Orion Nebula Cloud. The results of this study are presented and their implications discussed.

\section{The sample}

For WTTSs in the Chamaeleon, Corona Australis, Lupus, RhoOphiuchus, and Taurus SFRs, the sample used in the current paper to derive stellar parameters and chemical abundances was chosen in an identical manner to that in James et al. (2006), to which we refer the reader for extensive details. In brief, wTTS catalogues for each SFR were constructed from ROSAT All-Sky Survey [RASS] detections in and around each SFR. We then selected those stars having spectral types between $\mathrm{G}$ and early-K and visual magnitudes of 12 or brighter, which also have weak signatures of infrared excess and magnetic activity (e.g., having $\mathrm{H} \alpha$ equivalent widths $\ll 10 \AA$ ). We also restricted our sample to objects exhibiting substantial equivalent widths [EWs] of the lithium resonance line at $6708 \AA$ (whose substantial abundance is indicative of youth), as well as those stars which are not components of multiple systems, as judged from existing kinematic data.

In James et al. (2006), we presented the results of the analysis of a sub-sample of these stars, whose spectra were obtained using the FEROS spectrograph (2.2-m ESO/MPI telescope). The data were used to study the chemical abundances of WTTSs in 3 southern star-forming regions (Chamaeleon, $\mathrm{CrA}$ and Lupus). Unfortunately, the small aperture of the telescope did not allow us to obtain high $\mathrm{S} / \mathrm{N}$ data for that many interesting targets. The number of stars observed was thus quite low, and the $\mathrm{S} / \mathrm{N}$ of their spectra was often not of a quality sufficient to derive accurate stellar parameters and abundances. The use of a high resolution spectrograph installed on a larger telescope was thus mandatory to improve our results. In this paper, we present an extension of the study initiated in James et al. (2006), using new high S/N, high resolution spectroscopic observations, predominantly obtained using $8 \mathrm{~m}$-class telescopes.

After our new stellar spectra were reduced (see Sect. 3) we restricted a detailed spectroscopic analysis to narrow-lined stars, i.e., those having low projected equatorial rotational velocity $\left(<\sim 15 \mathrm{~km} \mathrm{~s}^{-1}\right)$. Calculations of projected equatorial rotational velocity $[v \sin i]$ for all stars were derived using the width of the cross-correlation function, using a method described by Melo et al. (2001; see also James et al. 2006). This is imperative because higher rotational velocities pose important limitations on the accuracy of derived stellar parameters and chemical abundances (see Sect. 4).

One-dimensional kinematic data (radial velocities [RVs]) of the target stars were compared with the expected systemic velocities of their parent SFR (using values calculated and compared in James et al. 2006). Target stars were only considered for a detailed spectroscopic abundance analysis if their RVs were invariant (1.e., not in a binary system) and they matched that of their natal cloud. A full description of the membership, mass and age properties of our target stars, as well as details on the rapid rotators, is presented in James et al. (in prep.).

For stars in the Orion Nebula Cloud, data and membership information were taken from the studies of Sicilia-Aguilar et al. (2005), Rhode et al. (2001), and Jones \& Walker (1988).

We note that one star from the Chamaeleon SFR (RX J1201.7-7959) whose metallicity was derived in James et al. (2006) is now excluded from the sample, because its RVs indicate that it is a spectroscopic binary system.

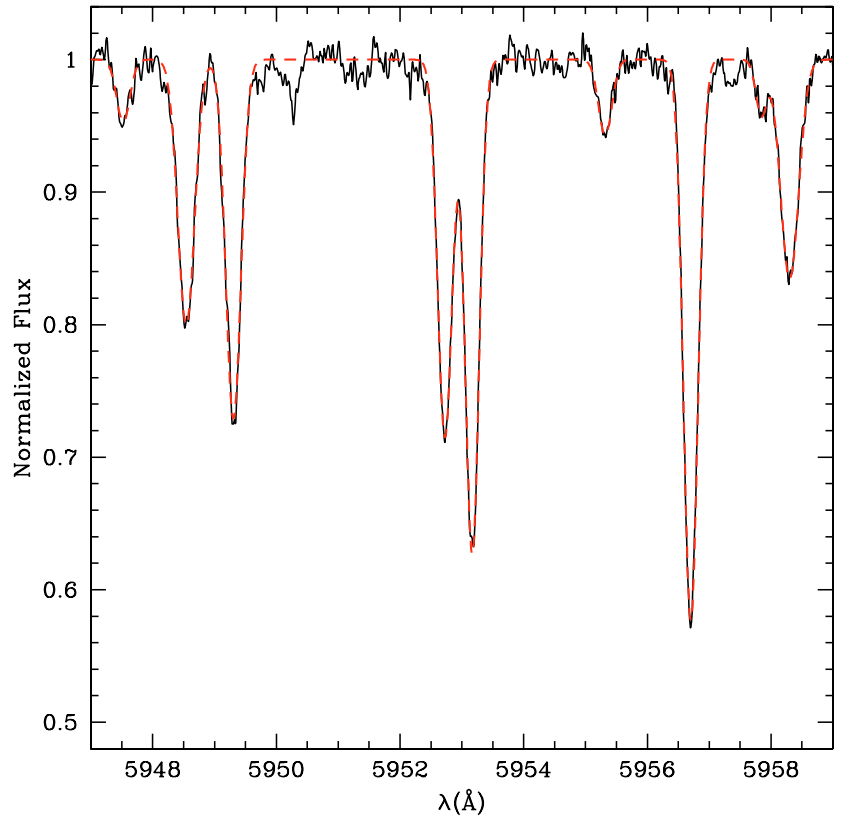

Fig. 1. Sample UVES spectrum for one of the stars in our sample (RX J1159.7-7601) in the region around $5950 \AA$. The spectrum presented has a $\mathrm{S} / \mathrm{N}$ ratio of $\sim 140$. Gaussian fits to most of the lines in the spectral domain presented are shown (dashed line). The EWs of the lines go from 12 to $130 \mathrm{~m} \AA$, the typical values for the Fe I lines in WTTS spectra used to derive stellar parameters and metallicity.

\section{Observations}

Between April and September 2005, we obtained a series of high resolution spectra for WTTSs in 4 young SFRs (Chamaeleon, Corona Australis, Lupus and Rho-Ophiuchus) using the UVES spectrograph on the VLT's Kueyen 8.2-m telescope (ESO program ID 075.C-0272). The spectra were obtained in Service Mode using the Red 580 mode, and cover the wavelength domain between 4800 and $7000 \AA$, with a gap between 5700 and $5850 \AA$. A binning of $2 \times 2$ pixels and a slit width of 0.9 arcsec was used, leading to a resolution of $R=\lambda / \Delta \lambda \sim 50000$. A sample UVES spectrum used is presented in Fig. 1.

To complement the UVES observations, six WTTSs in two more star-forming regions were observed. Observations of objects in the Orion Nebula Cloud [ONC] were carried out using the fibre link of the FLAMES-UVES spectrograph (ESO program ID 076.C-0524). The observations were carried out in Service Mode between October 2005 and March 2006. The CCD was used in a $1 \times 1$ binning mode, and the resultant spectra have a resolution of $\sim 50000$ and cover the same spectral domain as the UVES data. Finally, spectra for WTTS in the northern Taurus SFR were obtained in December 2006 using the SOPHIE fibrefed spectrograph at the 1.93-m telescope of the Observatoire de Haute Provence [OHP], France. The spectra were obtained in the High-Efficiency mode $(R \sim 40000)$, positioning one of the fibres on the object and the other on the sky. Background light subtraction was performed using the sky "spectrum", since the instrument data reduction software does not allow for the subtraction of inter-order scattered light.

For all data sets, spectral extraction and data reduction was achieved using the available instrument pipelines supplied by the observatories. Wavelength calibration was done using the spectrum of a thorium-argon lamp. The spectra were then Doppler corrected and set at a zero velocity. When more than one 
Table 1. List of WTTS observed in each SFR and derived stellar parameters, metallicities, and estimated $v \sin i$ values. The number of Fe I and Fe II lines used for the spectroscopic analysis is also listed, together with the RMS around the average abundance for each set of lines. Stellar parameters and $[\mathrm{Fe} / \mathrm{H}]$ derived from FEROS spectra ([4]) were taken from James et al. (2006).

\begin{tabular}{|c|c|c|c|c|c|c|c|c|}
\hline Star & $\begin{array}{l}T_{\text {eff }} \\
{[\mathrm{K}]}\end{array}$ & $\begin{array}{l}\log g_{\text {spec }} \\
{\left[\mathrm{cm} \mathrm{s}^{-2}\right]}\end{array}$ & $\begin{array}{c}\xi_{\mathrm{t}} \\
{\left[\mathrm{km} \mathrm{s}^{-1}\right]}\end{array}$ & {$[\mathrm{Fe} / \mathrm{H}]$} & Inst $^{\dagger}$ & $\begin{array}{c}N \\
(\mathrm{Fe} \mathrm{I}, \mathrm{II})\end{array}$ & $\begin{array}{c}\sigma \\
(\mathrm{Fe} \mathrm{I}, \mathrm{II}) \\
\end{array}$ & $\begin{array}{c}v \sin i \\
{\left[\mathrm{~km} \mathrm{~s}^{-1}\right]}\end{array}$ \\
\hline \multicolumn{9}{|l|}{ Cha } \\
\hline RX J1140.3-8321 & $4664 \pm 94$ & $4.17 \pm 0.52$ & $2.11 \pm 0.15$ & $-0.22 \pm 0.11$ & [1] & 31,4 & $0.11,0.29$ & 11 \\
\hline RX J1140.3-8321 & $4814 \pm 154$ & $4.07 \pm 0.92$ & $2.15 \pm 0.23$ & $-0.14 \pm 0.15$ & [4] & & & \\
\hline RX J1158.5-7754A & $4774 \pm 101$ & $4.08 \pm 0.41$ & $2.11 \pm 0.15$ & $-0.28 \pm 0.11$ & [1] & 29,5 & $0.10,0.21$ & 11 \\
\hline RX J1158.5-7754A & $4810 \pm 142$ & $3.87 \pm 0.51$ & $2.11 \pm 0.22$ & $-0.26 \pm 0.15$ & [4] & & & \\
\hline RX J1159.7-7601 & $4740 \pm 81$ & $4.37 \pm 0.33$ & $1.70 \pm 0.12$ & $-0.08 \pm 0.08$ & [1] & 32,5 & $0.08,0.17$ & 9 \\
\hline RX J1159.7-7601 & $4836 \pm 146$ & $4.23 \pm 0.33$ & $2.07 \pm 0.22$ & $-0.18 \pm 0.16$ & [4] & & & \\
\hline RX J1233.5-7523 & $5524 \pm 53$ & $4.49 \pm 0.16$ & $1.22 \pm 0.07$ & $-0.03 \pm 0.06$ & [1] & 35,11 & $0.06,0.07$ & 6 \\
\hline RX J1233.5-7523 & $5469 \pm 62$ & $4.40 \pm 0.22$ & $1.52 \pm 0.10$ & $-0.09 \pm 0.08$ & [4] & & & \\
\hline \multicolumn{9}{|l|}{$\mathrm{CrA}$} \\
\hline CrAPMS2 & $5400 \pm 164$ & $4.06 \pm 0.45$ & $2.41 \pm 0.30$ & $-0.09 \pm 0.18$ & [4] & & & \\
\hline CrAPMS-4SE & $5236 \pm 58$ & $4.33 \pm 0.17$ & $2.15 \pm 0.09$ & $-0.02 \pm 0.08$ & [1] & 35,8 & $0.07,0.08$ & 10 \\
\hline CrAPMS-4SE & $5335 \pm 101$ & $4.59 \pm 0.41$ & $2.24 \pm 0.17$ & $-0.02 \pm 0.12$ & [4] & & & \\
\hline RX J1839.0-3726 & $5150 \pm 66$ & $4.40 \pm 0.22$ & $2.45 \pm 0.11$ & $-0.10 \pm 0.08$ & [1] & 36,9 & $0.07,0.11$ & 10 \\
\hline RX J1839.0-3726 & $5293 \pm 127$ & $4.51 \pm 0.42$ & $2.19 \pm 0.21$ & $-0.01 \pm 0.15$ & [4] & & & \\
\hline \multicolumn{9}{|l|}{ Lup } \\
\hline RX J1507.2-3505 & $5169 \pm 63$ & $4.31 \pm 0.33$ & $2.09 \pm 0.09$ & $-0.06 \pm 0.08$ & [1] & 37,9 & $0.07,0.17$ & 13 \\
\hline RX J1507.2-3505 & $5155 \pm 148$ & $4.37 \pm 0.49$ & $2.53 \pm 0.26$ & $-0.08 \pm 0.14$ & [4] & & & \\
\hline RX J1546.6-3618 & $5223 \pm 83$ & $4.51 \pm 0.22$ & $2.22 \pm 0.12$ & $-0.04 \pm 0.09$ & [1] & 34,8 & $0.09,0.10$ & 6 \\
\hline RX J1546.6-3618 & $5062 \pm 109$ & $4.09 \pm 0.53$ & $2.12 \pm 0.16$ & $-0.12 \pm 0.14$ & [4] & & & \\
\hline RX J1547.6-4018 & $5006 \pm 61$ & $4.29 \pm 0.24$ & $1.87 \pm 0.08$ & $-0.06 \pm 0.07$ & [1] & 34,7 & $0.07,0.12$ & 12 \\
\hline RX J1547.6-4018 & $5045 \pm 120$ & $4.18 \pm 0.61$ & $2.23 \pm 0.20$ & $-0.14 \pm 0.13$ & [4] & & & \\
\hline RX J1601.1-3320 & $5479 \pm 152$ & $3.70 \pm 0.35$ & $2.49 \pm 0.26$ & $-0.05 \pm 0.18$ & [4] & & & \\
\hline RX J1523.4-4055 & $4923 \pm 87$ & $4.74 \pm 0.37$ & $2.05 \pm 0.16$ & $-0.03 \pm 0.09$ & [1] & 34,6 & $0.09,0.19$ & 10 \\
\hline \multicolumn{9}{|l|}{ Rho-Ohp } \\
\hline RX J1620.7-2348 & $4867 \pm 107$ & $4.40 \pm 0.29$ & $2.33 \pm 0.17$ & $-0.08 \pm 0.12$ & [1] & 35,7 & $0.12,0.13$ & 9 \\
\hline \multicolumn{9}{|l|}{ ONC } \\
\hline JW157 & $4877 \pm 83$ & $3.57 \pm 0.28$ & $1.97 \pm 0.09$ & $-0.06 \pm 0.11$ & [2] & 36,10 & $0.10,0.14$ & 7 \\
\hline JW589 & $4927 \pm 108$ & $3.90 \pm 0.30$ & $1.95 \pm 0.13$ & $-0.12 \pm 0.12$ & [2] & 33,5 & $0.11,0.15$ & 14 \\
\hline JW706 & $4599 \pm 79$ & $3.86 \pm 0.45$ & $1.50 \pm 0.10$ & $-0.17 \pm 0.09$ & [2] & 30,4 & $0.09,0.25$ & 12 \\
\hline \multicolumn{9}{|l|}{ Tau } \\
\hline 1RXSJ052146.7 & $4921 \pm 59$ & $4.05 \pm 0.29$ & $1.92 \pm 0.07$ & $-0.07 \pm 0.07$ & [3] & 34,5 & $0.07,0.15$ & 13 \\
\hline 1RXSJ053650.0 & $4918 \pm 112$ & $4.47 \pm 0.29$ & $1.96 \pm 0.17$ & $-0.18 \pm 0.10$ & [3] & 28,3 & $0.09,0.12$ & 14 \\
\hline 1RXSJ053931.0 & $5430 \pm 53$ & $4.09 \pm 0.23$ & $1.87 \pm 0.08$ & $0.05 \pm 0.07$ & [3] & 36,12 & $0.06,0.10$ & 11 \\
\hline
\end{tabular}

$\dagger$ Instruments used: [1] UVES; [2] FLAMES; [3] SOPHIE; [4] FEROS data from James et al. (2006).

spectrum of the same star was available, they were co-added. Final spectra have $\mathrm{S} / \mathrm{N}$ ratios between 100 and 200, and are of sufficient quality to derive stellar parameters and chemical abundances with a reasonable accuracy. In Table 1, we list fundamental physical properties for all the observed stars in each target SFR.

\section{Spectroscopic analysis}

\subsection{Stellar parameters and chemical abundances}

As in James et al. (2006), stellar parameters and iron abundances for the targets were derived in LTE, using a grid of plane-parallel, ATLAS 9 model atmospheres (Kurucz 1993) and the 2002 version of the radiative transfer code MOOG (Sneden 1973). The methodology used is described in detail in Santos et al. (2004, and references therein). The full spectroscopic analysis is based on the EWs of a set of Fe I and Fe II weak lines, by imposing ionization and excitation equilibrium, as well as a zero slope between the abundances given by individual lines and their equivalent width. The errors in the stellar parameters were derived using the same methodology as described in Gonzalez (1998).

For FGK dwarfs, the stellar parameters obtained using this methodology were shown to be compatible with other estimates in the literature. In particular, the effective temperatures derived are very close to the ones obtained using recent applications of the infra-red flux method (e.g. Casagrande et al. 2006).

The results of these analysis are presented in Table 1, where we also compare the values derived using UVES spectra for stars in common with the James et al. (2006) sample. We note that in their paper, James et al. used lower quality spectra taken with FEROS. A comparison of their results and our new ones shows that besides the larger error bars, the two datasets are in close agreement (well within error bars). For the duration of this paper, we will only consider the higher quality values derived from UVES spectra, for those stars having both FEROS and UVES measurements.

As was discovered in the studies by James et al. (2006) and (Padgett 1996), the derived micro-turbulence velocities are considerably above the ones found for main-sequence dwarfs with similar temperature detailed in the literature. The cause of these discrepancies is not yet clear to us, although the high values may be reflecting the effect of magnetic fields (Steenbock \& Holweger 1981).

Abundances for silicon (alpha-element) and nickel (ironpeak element) were derived using the methodology and atomic line lists described in Gilli et al. (2006) and Santos et al. (2006), and are listed in Table2. Analysis of these two elements was 
Table 2. Abundances of $\mathrm{Si}$ and $\mathrm{Ni}$ for the stars in Table 1 that were observed with UVES, FLAMES or SOPHIE spectrographs. The [Fe/H] values listed in Table 1 are also presented for comparison.

\begin{tabular}{lccccccc}
\hline \hline Star & {$[\mathrm{Fe} / \mathrm{H}]$} & {$[\mathrm{Si} / \mathrm{H}]$} & $\sigma([\mathrm{Si} / \mathrm{H}])$ & $\mathrm{N}(\mathrm{Si})$ & {$[\mathrm{Ni} / \mathrm{H}]$} & $\sigma([\mathrm{Ni} / \mathrm{H}])$ & $\mathrm{N}(\mathrm{Ni})$ \\
\hline Cha & & & & & & & \\
RX J1140.3-8321 & -0.22 & -0.19 & 0.08 & 4 & -0.30 & 0.13 & 15 \\
RX J1158.5-7754A & -0.28 & -0.27 & 0.07 & 4 & -0.34 & 0.14 & 14 \\
RX J1159.7-7601 & -0.08 & -0.05 & 0.07 & 5 & -0.13 & 0.09 & 18 \\
RX J1233.5-7523 & -0.03 & 0.00 & 0.04 & 9 & -0.07 & 0.04 & 18 \\
\hline CrA & & & & & & & \\
CrAPMS-4SE & -0.02 & -0.01 & 0.07 & 9 & -0.08 & 0.13 & 18 \\
RX J1839.0-3726 & -0.10 & -0.10 & 0.10 & 8 & -0.22 & 0.11 & 16 \\
\hline Lup & & & & & & & \\
RX J1507.2-3505 & -0.06 & -0.02 & 0.09 & 8 & -0.18 & 0.08 & 14 \\
RX J1523.4-4055 & -0.03 & -0.01 & 0.10 & 6 & -0.13 & 0.11 & 14 \\
RX J1546.6-3618 & -0.04 & -0.05 & 0.07 & 9 & -0.11 & 0.10 & 18 \\
RX J1547.6-4018 & -0.06 & -0.03 & 0.09 & 8 & -0.15 & 0.12 & 12 \\
\hline Rho-Oph & & & & & & & \\
RX J1620.7-2348 & -0.08 & -0.04 & 0.16 & 6 & -0.12 & 0.12 & 17 \\
\hline ONC & & & & & & & \\
JW147 & -0.06 & -0.07 & 0.12 & 9 & -0.18 & 0.07 & 21 \\
JW589 & -0.12 & -0.20 & 0.12 & 4 & -0.18 & 0.09 & 11 \\
JW706 & -0.17 & -0.07 & 0.13 & 6 & -0.16 & 0.08 & 13 \\
\hline Tau & & & & & & & \\
1RXSJ052146.7 & -0.07 & 0.01 & 0.08 & 7 & -0.12 & 0.13 & 11 \\
1RXSJ053650.0 & -0.18 & -0.14 & 0.11 & 4 & -0.23 & 0.13 & 11 \\
1RXSJ053931.0 & 0.05 & 0.05 & 0.09 & 10 & -0.03 & 0.09 & 13 \\
\hline
\end{tabular}

Table 3. Weighted average and dispersion of the metallicities and $[\mathrm{X} / \mathrm{Fe}](\mathrm{X}=\mathrm{Si}, \mathrm{Ni})$ abundance ratios derived for each $\mathrm{SFR} . N$ and $N^{\prime}$ are the number of stars used for the determination of $\langle[\mathrm{Fe} / \mathrm{H}]\rangle$ and $\langle[\mathrm{X} / \mathrm{H}]\rangle$, respectively. For Rho-Oph, since only one star was measured, the RMS of the values correspond to the rms of the abundance determination for the star.

\begin{tabular}{lcccccccc}
\hline \hline SFR & $\langle[\mathrm{Fe} / \mathrm{H}]\rangle$ & $\mathrm{rms}$ & $N$ & $\langle[\mathrm{Si} / \mathrm{Fe}]\rangle$ & $\sigma([\mathrm{Si} / \mathrm{Fe}])$ & $\langle[\mathrm{Ni} / \mathrm{Fe}]\rangle$ & $\sigma([\mathrm{Ni} / \mathrm{Fe}])$ & $N^{\prime}$ \\
\hline Cha & -0.11 & 0.11 & 4 & 0.03 & 0.01 & -0.05 & 0.02 & 4 \\
CrA & -0.06 & 0.05 & 3 & 0.01 & 0.01 & -0.09 & 0.04 & 2 \\
Lup & -0.05 & 0.01 & 5 & 0.02 & 0.02 & -0.10 & 0.02 & 4 \\
Rho-Oph & -0.08 & 0.12 & 1 & 0.04 & 0.16 & -0.04 & 0.12 & 1 \\
ONC & -0.13 & 0.06 & 3 & 0.00 & 0.09 & -0.06 & 0.07 & 3 \\
Tau & -0.05 & 0.11 & 3 & 0.04 & 0.04 & -0.07 & 0.02 & 3 \\
\hline
\end{tabular}

performed because they have several well defined lines in the spectra of solar type stars, and because they do not present any strong dependence of derived abundance with effective temperature (Bodaghee et al. 2003; Gilli et al. 2006). This increases our confidence of uniformity in the comparison with abundances derived for solar-type field stars, presented in Gilli et al. (2006).

\subsection{Veiling}

The spectroscopic analysis described above relies on the measurement of EWs for a set of Fe I and Fe II lines. This means that any contribution of veiling, related to stellar accretion phenomena, could lead to erroneous values for the stellar parameters and effective temperatures. Although high levels of veiling are not expected for the WTTS in our sample (Hartigan et al. 1995), we err on the side of caution, and have performed a more detailed analysis.

The veiling (defined as the ratio between the excess continuum flux and the photospheric continuum contribution to the spectrum) was derived using a method based on that described in Hartigan et al. (1989). With this method, the line depth of photospheric absorption lines are compared with a reference spectrum within a small wavelength band (a few tens of $\AA$ wide), and it is assumed that the veiling be constant in this region. The wavelength coverage of our spectra is almost complete from 4800 to $7000 \AA$ with a small gap between 5700 and $5850 \AA$. For each object, a reference star (with similar temperature as the object) was rotationally broadened to match the $v \sin i$ of the object, and the veiling computed in intervals of $20 \AA$. The reference stars are dwarfs of similar effective temperature and metallicity to the targets, and were the ones used in previous chemical abundances studies of stars with and without extra-solar planets (Santos et al. 2004, 2005; Sousa et al. 2006).

No clear trend in the veiling variation with wavelength was found for any of the stars. For all the objects, the veiling is centred near zero with some scatter (Fig. 2). The values displayed in Table 4 represent the mean veiling bluewards of $5700 \AA$ and redwards of $5850 \AA$. The error bars represent the standard deviation in each region. The negative values and the highest error bars found in the red region are due to shallower photospheric lines and the presence of telluric lines in the object spectra, which are poorly fitted by the spectra of the rotationally broadened reference stars.

\section{Results and discussion}

\subsection{Systematic errors or an abundance dispersion inside the SFR}

As can be seen from Tables 1 and 3, the general dispersion observed in the $[\mathrm{Fe} / \mathrm{H}]$ values of the stars in each SFR is quite small. The same is true for the abundances of $\mathrm{Si}$ and Ni (Table 2 and Fig. 3). Possible exception is the Chamaeleon SFR, where 


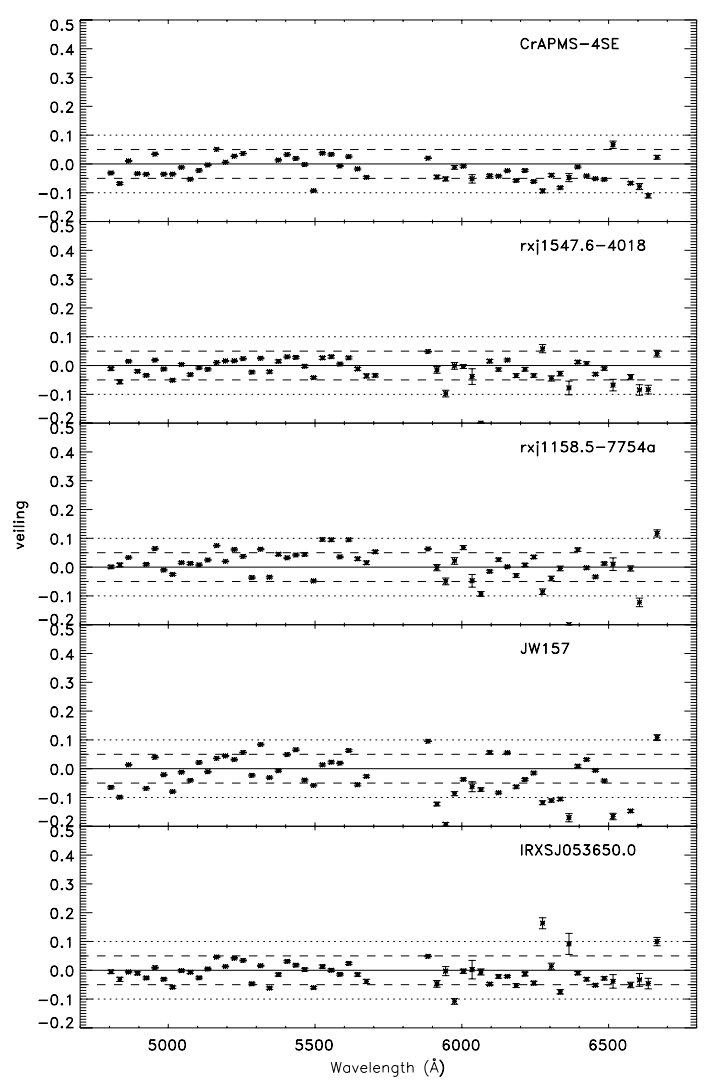

Fig. 2. Dependence of veiling with wavelength for five stars in our sample with different temperatures. The dashed and dotted lines show, respectively, the veiling at levels \pm 0.05 and \pm 0.10 . Negative veiling occurs when the photospheric lines in the reference star are shallower than in the target.

the dispersion of the measured metallicities is quite high for the 4 stars studied (although still of the order of magnitude of the abundance uncertainties). Moreover, the abundances of the three species studied seem to be correlated, in the sense that higher iron abundances also correspond to higher $[\mathrm{Si} / \mathrm{H}]$ and $[\mathrm{Ni} / \mathrm{H}]$ values.

A look at the temperature and metallicity values in Table 1 suggests, however, that a correlation may exist between metallicity and effective temperature. Using all 19 stars in our sample, we find a slope of $\sim 0.15 \mathrm{dex} / 1000 \mathrm{~K}$ for the points in a temperature-metallicity plot (in this fit, $[\mathrm{Fe} / \mathrm{H}]=0.0$ would occur for $T_{\text {eff }} \sim 5500 \mathrm{~K}$ ). Although it may be unwise to use stars from different SFRs to conclude, this trend may suggest the existence of systematic errors in the derived stellar parameters.

For a fixed metallicity, lower temperature stars tend to have deeper spectral lines. The strongest of these may have extended damping wings. The fitting of gaussian profiles to measure the line-EWs could thus under-estimate the measurements, leading to lower metallicity estimates. Although this hypothesis could in principle explain the observed correlation, a look at individual lines in our spectra (Fig. 1) shows that in the range of EWs measured, the observed spectral lines are well approximated by Gaussian functions. This is actually expected, given that the large majority of the lines used have EWs below 100-150 m $\AA$.

Furthermore, no correlation is seen in the upper envelope of the temperature-metallicity plot of the large sample of stars presented in Santos et al. (2004), whose parameters were obtained using the same line-lists and model atmospheres. We can add that the metallicities and efective temperatures derived for main
Table 4. Veiling determinations for the stars listed in Table 1 that were observed with UVES, FLAMES or SOPHIE spectrographs.

\begin{tabular}{lcc}
\hline \hline Star & Veil & Veil \\
& $<5700 \AA$ & $>5850 \AA$ \\
\hline Cha & & \\
RX J1140.3-8321 & $-0.01 \pm 0.04$ & $-0.07 \pm 0.06$ \\
RX J1158.5-7754A & $0.03 \pm 0.04$ & $0.00 \pm 0.05$ \\
RX J1159.7-7601 & $-0.01 \pm 0.03$ & $-0.05 \pm 0.06$ \\
RX J1233.5-7523 & $0.02 \pm 0.01$ & $0.04 \pm 0.02$ \\
\hline CrA & & \\
CrAPMS-4SE & $0.00 \pm 0.04$ & $-0.04 \pm 0.04$ \\
RX J1839.0-3726 & $0.00 \pm 0.05$ & $-0.05 \pm 0.05$ \\
\hline Lup & & \\
RX J1507.2-3505 & $0.00 \pm 0.05$ & $-0.03 \pm 0.05$ \\
RX J1546.6-3618 & $-0.01 \pm 0.04$ & $-0.05 \pm 0.05$ \\
RX J1547.6-4018 & $0.00 \pm 0.03$ & $-0.01 \pm 0.04$ \\
RX J1523.4-4055 & $-0.02 \pm 0.06$ & $-0.07 \pm 0.07$ \\
\hline Rho-Ohp & & \\
RX J1620.7-2348 & $0.01 \pm 0.04$ & $-0.04 \pm 0.06$ \\
\hline ONC & & \\
JW157 & $0.00 \pm 0.05$ & $-0.06 \pm 0.08$ \\
JW589 & $0.02 \pm 0.10$ & $-0.04 \pm 0.08$ \\
JW706 & & $-0.03 \pm 0.06$ \\
\hline Tau & $0.00 \pm 0.04$ & $-0.03 \pm 0.06$ \\
1RXSJ052146.7 & $0.00 \pm 0.03$ & $-0.02 \pm 0.05$ \\
1RXSJ053650.0 & $0.04 \pm 0.05$ & $0.00 \pm 0.05$ \\
1RXSJ053931.0 & &
\end{tabular}

sequence dwarfs using the methodology presented here are in good agreement with those found in the literature, derived using other methods and model atmospheres (e.g. Santos et al. 2004; Casagrande et al. 2006).

Morel et al. (2004) studied the detailed effects of stellar spots affecting the determination of stellar parameters and metallicity for RS CVn stars. Their results show that the final abundances can be affected, although not always strongly, by the presence of photospheric spots. This issue may have particular importance when studying young, magnetically active stars like the ones in our sample. These effects possibly contribute to the observed metallicity-temperature correlation.

We have also verified the possibility that a small residual veiling is causing the observed differences. For this purpose, we performed a simple test by increasing 3\% the measured EW of iron lines used to derive stellar parameters for the two most metal deficient stars in the the Cha SFR (RX J1140.3-8321, RX $\mathrm{J} 1158.5-7754 \mathrm{a})$. The value of $3 \%$ is actually a conservative upper limit for the observed veiling errors (note that the uncertainties listed in Table 4 represent the observed rms in the different veiling points, and not the error on the average, which is much smaller). The resulting stellar parameters show only very small variations (below $10 \mathrm{~K}$ in temperature and $0.01 \mathrm{dex}$ in surface gravity), and their iron abundances increased by a mere 0.02 dex. Clearly, small amounts of un-accounted veiling cannot explain the observed abundance dispersion.

In any case, current data suggests that the observed dispersions may be the result of some un-identified systematic effect in our data, and not to the existence of a real dispersion in the metallicities inside each SFR. This conclusion also implies that the observed dispersions must be seen as upper limits.

\subsection{Other elements}

Chemical abundances of several elements are useful in distinguishing between different populations in the Galaxy (e.g. 


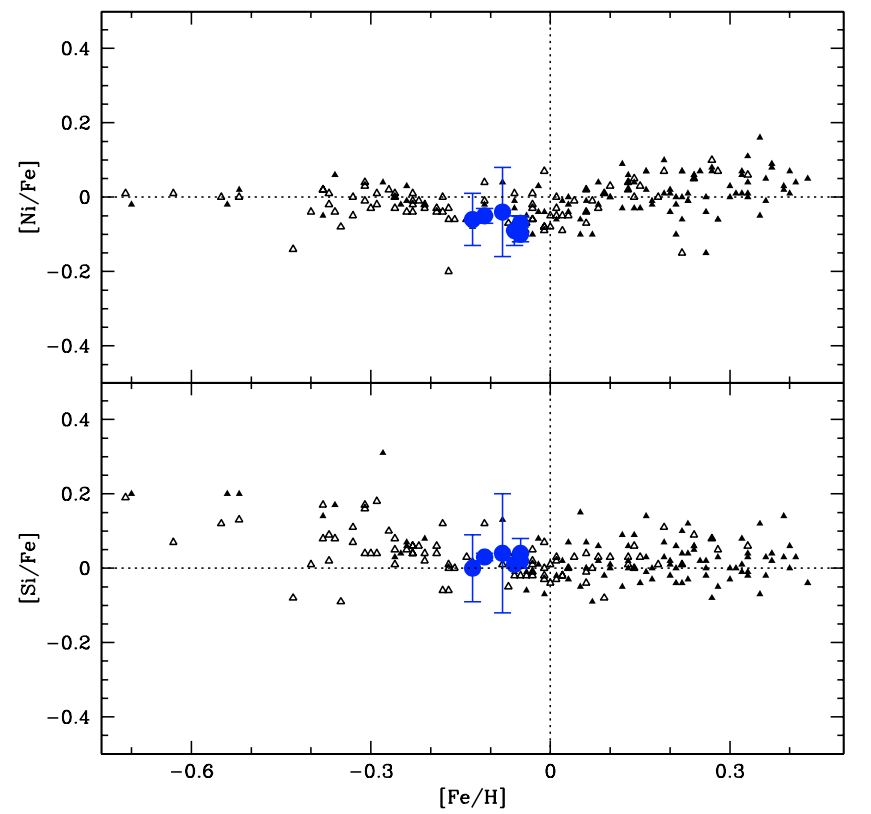

Fig. 3. Abundance ratios $[\mathrm{Si} / \mathrm{Fe}]$ and $[\mathrm{Ni} / \mathrm{Fe}]$ as a function of $[\mathrm{Fe} / \mathrm{H}]$ for the six SFR (large circles) when compared with the same ratios for planet-host stars (filled triangles) and "single" stars (open triangles) from Gilli et al. (2006). The dotted lines mark the solar abundances.

Nissen 2004; Fuhrmann 2004; Bensby et al. 2005; Brewer \& Carney 2006; Rich \& Origlia 2005; Fulbright et al. 2005). The observed differences are meant to reflect different star formation histories or stellar initial mass functions in the different Galactic systems (Carigi 2006). It is thus interesting to check whether the abundances of the elements studied in the current paper for the six SFRs are different from those found in field disk stars.

In Fig. 3 we compare the abundance ratios $[\mathrm{Si} / \mathrm{Fe}]$ and $[\mathrm{Ni} / \mathrm{Fe}]$ listed in Table 3 with those found for field planet-host stars and "single" stars from Gilli et al. (2006). We remind the reader that Gilli et al. used the same procedure used in the current paper to derive the abundances of these elements (same model atmospheres, atomic line lists, etc.), rendering such a comparison free from important systematic errors.

The results show that no clear differences exist between the abundance ratios of the studied SFRs and those of field stars of the same metallicity. The chemical abundances in nearby SFRs seem to reflect those of typical, thin disk stars.

\subsection{The metallicity distribution of nearby SFRS}

In Table 3, we present the weighted average metallicities and abundance ratios for each SFR, derived from the values listed in Table 1. In all cases the average abundances found are below solar. The metallicities for the six SFRs vary from -0.13 (ONC) to -0.05 (Lupus and Taurus). Intriguingly, this result suggests that metal-rich star-forming clouds in the solar neighbourhood are rare. The implications of this result are discussed in Sects. 5.3.2 and 5.3.3.

In Fig. 4 we compare the metallicity distribution of our sample of six SFRs (dashed line) with the same distribution for 1250 stars in the volume-limited CORALIE planet-search sample (see Udry et al. 2000; Santos et al. 2004). The two distributions have similar average metallicities $(-0.08$ for the SFR

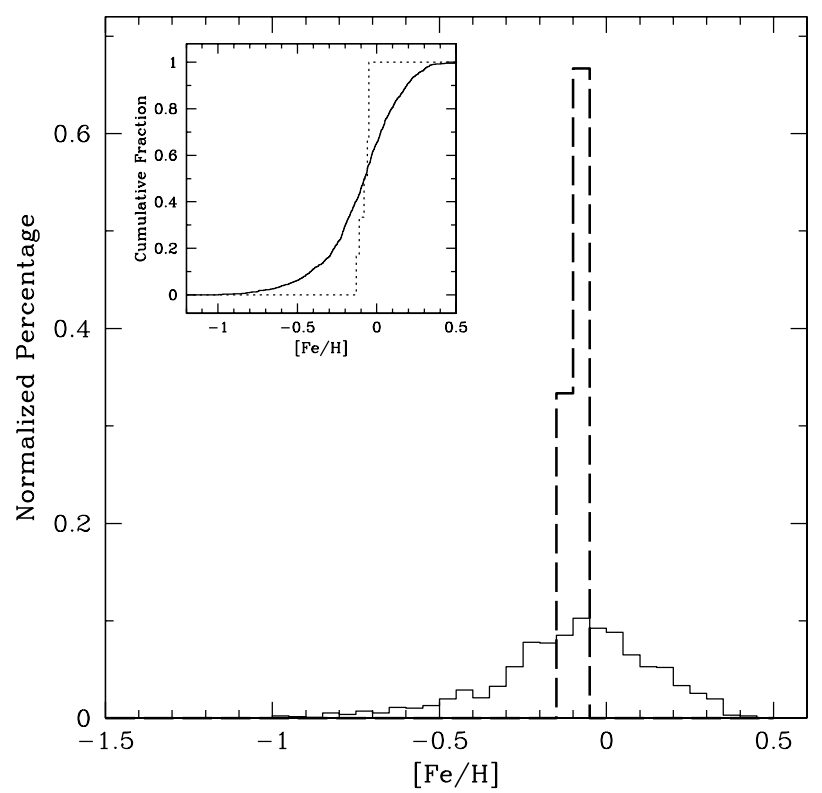

Fig. 4. Metallicity distribution of solar-neighbourhood stars in a volume-limited sample (filled line - see text) and of the metallicities of the six SFRs analyzed in this paper (dashed line). In inset we show the cumulative functions of the two distributions.

sample, and -0.10 for the field stars). However, the metallicity distribution of the stars in the solar neighbourhood is clearly wider $(\sigma=0.24 \mathrm{dex})$, when compared with the one of the SFRs $(\sigma=0.033 \mathrm{dex})^{2}$.

To have some insight into the statistical meaning of the observed difference, we performed two different tests. First, a simple Kolmogorov-Smirnov test was done to verify the possibility that both samples belong to the same population. The result shows that $P(\mathrm{KS}) \sim 14 \%$, a value that is clearly not significant, likely due to the small number of the observed SFRs.

To better settle these issues we then performed a series of Monte-Carlo simulations. These were done to test the probability that in any given sample of randomly selected six metallicity points (taken from the field star sample) we would have a distribution similar to the one observed for the SFRs. In the simulation, the metallicity values were computed taking into account the observed metallicity distribution for field stars presented in Fig. 4. The methodology used is similar to the one presented in Udry et al. (2003).

A total of 100000 data sets were simulated, and for each of them we computed the values for $\sigma([\mathrm{Fe} / \mathrm{H}])$ of the six simulated points. We then compared the number of cases where the simulated $\sigma([\mathrm{Fe} / \mathrm{H}])$ is smaller than the observed one $(0.033 \mathrm{dex})$.

The results of these simulations show that the very small $[\mathrm{Fe} / \mathrm{H}]$ dispersion observed is clearly at odds with the expected metallicity distribution of solar-type field stars. In our simulations, in only $0.02 \%$ of the cases we obtained a value of $\sigma([\mathrm{Fe} / \mathrm{H}])$ lower than the observed 0.033 dex (Fig. 5). In other words, the very small dispersion observed for the metallicity distribution of the observed SFRs is clearly significant.

\footnotetext{
2 The average metallicity and dispersion of the field star sample is the same as the one found in the metallicity distribution for the subset of 94 CORALIE sample stars with high precision metallicity estimates from Santos et al. (2005).
} 


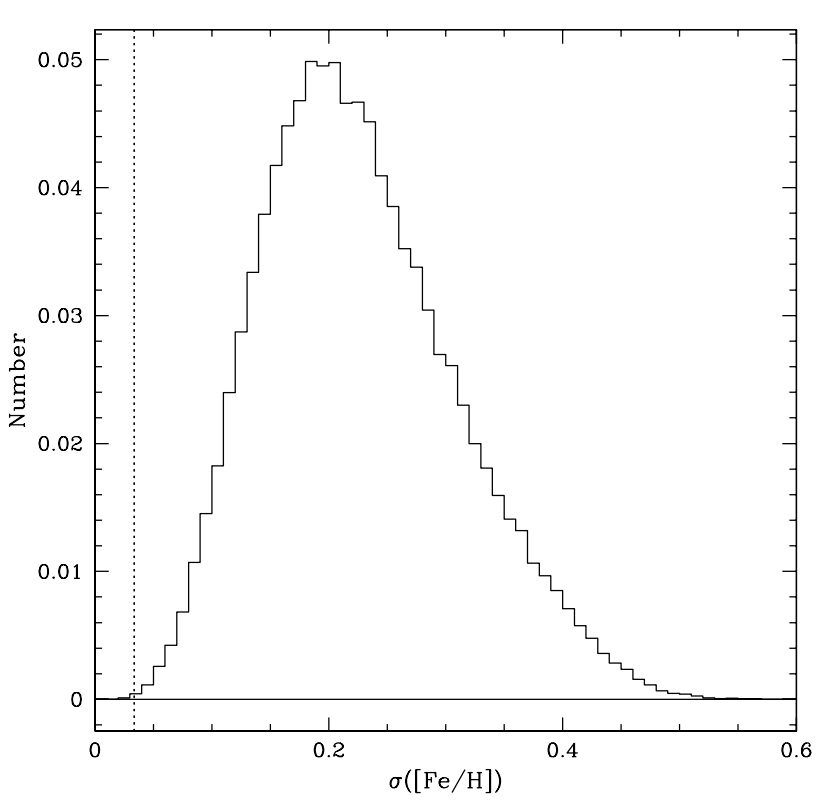

Fig. 5. Distribution of 100000 simulated $[\mathrm{Fe} / \mathrm{H}]$ dispersions (RMS). The dotted line denotes the observed RMS of the 6 star-forming regions. In only $0.02 \%$ of the cases we obtained a dispersion below the observed value $(0.033 \mathrm{dex})$.

\subsubsection{The small metallicity dispersion}

The small metallicity dispersion observed for the six starforming regions $(0.033 \mathrm{dex})$ agrees with other recent studies done by Nieva \& Przybilla (2006), who derived similar values for the dispersion of the abundances in nearby OB stars (0.04 dex). When compared with local field dwarfs, a small dispersion is also observed in the metallicities of open star clusters (Twarog et al. 1997), nearby young FGK stars and nebular abundances (see compilation by Sofia \& Meyer 2001).

Together with the literature results, the data presented in this paper suggests that the material in the nearby inter-stellar medium [ISM] is very uniform. In turn, this suggests that at any given Galacto-centric radius, the ISM is likely to be well mixed, and that mixing timescales are very short when compared with the time between different enrichment events (see discussion in Roy \& Kunth 1995; Wielen et al. 1996). At a given epoch and Galacto-centric radius, the chemical abundances in the ISM are likely to be uniform.

If we consider that nearby SFRs are good representatives of the current metallicity in the solar circle, the small scatter and slightly lower than solar abundances observed further imply that the metallicity distribution of main-sequence stars in the solar neighbourhood (with a much larger metallicity dispersion) is strongly affected by stellar diffusion (e.g. Wielen et al. 1996).

\subsubsection{The sub-solar metallicity of SFRS}

Although the average metallicity of the observed SFRs is below solar, in Sect.5.1 we have seen that some dependence seems to exist between the measured metallicities and effective temperatures (a positive gradient of $\sim 0.15 \mathrm{dex} / 1000 \mathrm{~K}$ is observed). Since our stars are all cooler than the Sun $\left(4664<T_{\text {eff }}<\right.$ $5524 \mathrm{~K}$ ), this could imply that some under-estimate of the metallicities is done in our parameter estimate. We cannot exclude that the average metallicity of the observed SFRs may be closer to solar, although we can reasonably exclude a value that is strongly above.

Our results thus do not exclude (and somehow support) some models of Galacto-chemical evolution (Chiappini et al. 2003; Carigi et al. 2005) that predict that a slow chemical enrichment has happened in the Galactic disk during the last 4.5 Gyr. We should add, however, that models of chemical evolution of the galaxy often talk about oxygen as metallicity proxy, while in the current paper we measured iron abundances. Contrarily to oxygen, iron is formed in SN Ia events, that are supposed to continue even once the star-forming rate has slowed down. It will thus be interesting to explore the impact of our results on the models of galactic chemical evolution.

If, on the other hand, the sub-solar abundances observed are real (or at least if the abundances are lower than those expected by models), the results could fit well a scenario where the Sun was formed in an inner region of the Milky-Way's disk (later on experiencing orbital diffusion - Wielen et al. 1996; Edvardsson et al. 1993), or that the nearby ISM suffered from a recent in-fall of metal-poor gas ${ }^{3}$. Sub-solar abundances were recently found for oxygen and carbon in B-type stars (Nieva \& Przybilla 2006) and in the local bubble (Oliveira et al. 2005), although in the latter case dust depletion may account for the difference. These sub-solar metallicities are however at odds with some other studies of chemical abundances in H II regions (Esteban et al. 2004, 2005).

Recent values for the solar oxygen abundance (lower than previously considered) also suggest that the ISM abundances may be closer to solar than previously thought (e.g. Chiappini et al. 2003; Asplund et al. 2004). A recent review on this question is addressed by Allende Prieto (2007). However, if we consider that heavy element settling has occurred in the Sun (Lodders 2003; Bahcall et al. 2006) decreasing its abundance with respect to the initial value, the lower-abundances problem can be even more important than suggested by the mentioned values.

\subsubsection{On the origin of metal-rich stars}

As mentioned above, current models of galactic chemical evolution predict that a slow chemical enrichment has happened in the galactic disk during the past 4.5 Gyr. Indeed, the models cannot easily explain the existence of super metal-rich stars in the solar neighbourhood.

Our results thus support the idea that super metal-rich stars (with metallicity clearly above solar) found in the solar vicinity may have been formed in inner Galactic disk regions. In a recent paper, Ecuvillon et al. (2007) present evidence that nearby metal-rich stars have kinematics similar to those of the Hyades (metal-rich) stream (see e.g. Famaey et al. 2007). This is also the case of stars with planetary-mass companions, that are found to be metal-rich when compared with single field stars (Santos et al. 2004; Ecuvillon et al. 2007). Famaey et al. (2007) and Ecuvillon et al. (2007) interpret these results on the basis of a scenario in which metal-rich stars in the solar neighbourhood (and in particular planet-hosts) could have formed in a more metal rich ISM and then suffered radial displacements due to a non-axisymmetric component of the Galactic potential, pushing them into the solar neighbourhood (see also Grenon 1999).

\footnotetext{
3 Although this latter hypothesis seems unlikely given the large quantities of printine gas needed (see Carigi et al. 2006).
} 
In this sense, it is interesting to see that for many chemical elements the abundance ratios $[\mathrm{X} / \mathrm{Fe}]$ seem to present a rising trend as a function of $[\mathrm{Fe} / \mathrm{H}]$ in the metal-rich domain, i.e., for $[\mathrm{Fe} / \mathrm{H}]>0.0$ (Bensby et al. 2005; Gilli et al. 2006). Such trends seem very difficult to explain with current models of galactic chemical evolution (Carigi 2006). Curiously, for a given $[\mathrm{Fe} / \mathrm{H}]$, higher abundance ratios are also observed for stars in other galactic populations (e.g. Fuhrmann 2004; Zoccali et al. 2006; Rich \& Origlia 2005).

Also interestingly, there are suggestions that lithium abundances in planet-hosting, metal-rich stars show a systematic difference with respect to field stars in the temperature range between 5600 and $5850 \mathrm{~K}$ (Israelian et al. 2004; Chen \& Zhao 2006). We can speculate that the explanation for this difference can be related to a different lithium evolution in the inner Galactic disk.

\section{Concluding remarks}

In this paper, we present stellar metallicities for six nearby SFRs, derived from high resolution spectroscopy of young WTTSs. The results show that all the studied SFRs have metallicities between -0.13 and -0.05 dex, with a very small scatter of 0.033 dex around the mean value of $\langle[\mathrm{Fe} / \mathrm{H}]\rangle=-0.08 \mathrm{dex}$ (compatible to solar within the uncertainties).

The analysis of the abundances of silicon (alpha-element) and nickel (iron-peak element) also suggests that the abundance ratios in the studied SFRs are typical of those found in solar neighbourhood thin disk stars of similar metallicity.

Together with other results from the literature, these observations suggest that the chemical abundances in the nearby ISM are extremely uniform and not strongly above solar. Such a conclusion may have important implications for the models of Galactic evolution.

The results presented here have also important implications on planet searches around young (active) stars. Although these searches are severely limited in current radial-velocity surveys (e.g. Saar \& Donahue 1997; Santos et al. 2000; Paulson et al. $2002)^{4}$, several instruments are now being built that will change this situation. In particular, new generation adaptive optics systems like the ESO SPHERE planet finder or the Gemini GPI project, as well as a new generation of interferometric instruments like PRIMA (ESO), will allow us to study, in unprecedented detail, the existence of long period planets around young solar-type stars. The rarity of metal-rich nearby star-forming regions may limit the goals of these projects if the metallicity-giant planet connection is still present for systems with long orbital periods.

In this context it will also be very interesting to study in detail the abundances of nearby young, post-T Tauri stars (e.g. Zuckerman \& Song 2004; Torres et al. 2006). Such studies would also help to settle the question about the metallicity of the local ISM (e.g. Sofia \& Meyer 2001).

If stars with planets have originated from inner galactic regions, it may be interesting to understand how planetary evolution (and survival) may suffer with stellar orbital diffusion. This conclusion may further have impact on the understanding of the frequency of planets in the Galaxy.

As a very important by-product, the observations presented in this paper can further be used to investigate the effects of the metallicity on the properties of stellar populations such as

\footnotetext{
${ }^{4}$ See however recent detection by Setiawan et al. (2008).
}

rotation, multiplicity degree, or magnetic activity. These studies will be presented in a separate paper, James et al. (in prep.).

Acknowledgements. We would like to thank M. F. Nieva, L. Carigi, C. Chiappini, and G. Carraro for the fruitful discussions. We also thank the comments by the anonymous referee who helped to improve the paper. N.C.S. acknowledges the support from Fundação para a Ciência e a Tecnologia (FCT), Portugal, in the form of a grant (references POCI/CTE-AST/56453/2004 and PPCDT/CTE-AST/56453/2004). This work was supported in part by the EC's FP6 and by FCT (with POCI2010 and FEDER funds), within the HELAS international collaboration. JFG acknowleges the support from FCT (project PTDC/CTE-AST/65971/2006). We [D.J.J.] gratefully acknowledge financial support from the Programme National de Physique Stellaire de l'INSU for observations at OHP, and by support from an NSF grant [AST-0349075] to Vanderbilt University.

\section{References}

Allende Prieto, C. 2007, [arXiv:astro-ph/0702429]

Asplund, M., Grevesse, N., Sauval, A. J., Allende Prieto, C., \& Kiselman, D. 2004, A\&A, 417, 751

Bahcall, J. N., Serenelli, A. M., \& Basu, S. 2006, ApJS, 165, 400

Bensby, T., Feltzing, S., Lundström, I., \& Ilyin, I. 2005, A\&A, 433, 185

Benz, W., Mordasini, C., Alibert, Y., \& Naef, D. 2006, in Tenth Anniversary of 51 Peg-b: Status of and prospects for hot Jupiter studies, ed. L. Arnold, F. Bouchy, \& C. Moutou, 24

Bodaghee, A., Santos, N. C., Israelian, G., \& Mayor, M. 2003, A\&A, 404, 715 Brewer, M.-M., \& Carney, B. W. 2006, AJ, 131, 431

Carigi, L. 2006, [arXiv: astro-ph/0612049]

Carigi, L., Peimbert, M., Esteban, C., \& García-Rojas, J. 2005, ApJ, 623, 213

Carigi, L., Colín, P., \& Peimbert, M. 2006, ApJ, 644, 924

Casagrande, L., Portinari, L., \& Flynn, C. 2006, MNRAS, 373, 13

Chen, Y. Q., \& Zhao, G. 2006, AJ, 131, 1816

Chiappini, C., Romano, D., \& Matteucci, F. 2003, MNRAS, 339, 63

Ecuvillon, A., Israelian, G., Pont, F., Santos, N. C., \& Mayor, M. 2007, A\&A, 461,171

Edvardsson, B., Andersen, J., Gustafsson, B., et al. 1993, A\&A, 275, 101

Esteban, C., Peimbert, M., García-Rojas, J., et al. 2004, MNRAS, 355, 229

Esteban, C., García-Rojas, J., Peimbert, M., et al. 2005, ApJ, 618, L95

Famaey, B., Pont, F., Luri, X., et al. 2007, A\&A, 461, 957

Fischer, D. A., \& Valenti, J. 2005, ApJ, 622, 1102

Fuhrmann, K. 2004, Astron. Nachr., 325, 3

Fulbright, J. P., McWilliam, A., \& Rich, R. M. 2005, Am. Astron. Soc. Meet. Abstr., 207, 133.04

Gilli, G., Israelian, G., Ecuvillon, A., Santos, N. C., \& Mayor, M. 2006, A\&A, 449,723

Gonzalez, G. 1997, MNRAS, 285, 403

Gonzalez, G. 1998, A\&A, 334, 221

Gonzalez, G., Laws, C., Tyagi, S., \& Reddy, B. E. 2001, AJ, 121, 432

Grenon, M. 1999, Ap\&SS, 265, 331

Hartigan, P., Hartmann, L., Kenyon, S., Hewett, R., \& Stauffer, J. 1989, ApJS, 70,899

Hartigan, P., Edwards, S., \& Ghandour, L. 1995, ApJ, 452, 736

Haywood, M. 2006, MNRAS, 371, 1760

Ida, S., \& Lin, D. N. C. 2004, ApJ, 616, 567

Israelian, G., Santos, N. C., Mayor, M., \& Rebolo, R. 2004, A\&A, 414, 601

James, D. J., Melo, C., Santos, N. C., \& Bouvier, J. 2006, A\&A, 446, 971

Jones, B. F., \& Walker, M. F. 1988, AJ, 95, 1755

Kurucz, R. 1993, ATLAS9 Stellar Atmosphere Programs and 2 km/s grid, Kurucz CD-ROM No. 13, Cambridge, Mass.: Smithsonian

Astrophysical Observatory, 13

Lodders, K. 2003, ApJ, 591, 1220

Melo, C. H. F., Pasquini, L., \& De Medeiros, J. R. 2001, A\&A, 375, 851

Morel, T., Micela, G., Favata, F., \& Katz, D. 2004, A\&A, 426, 1007

Murray, N., Hansen, B., Holman, M., \& Tremaine, S. 1998, Science, 279, 69

Nieva, M. F., \& Przybilla, N. 2006, ApJ, 639, L39

Nissen, P. E. 2004, in Origin and Evolution of the Elements, ed. A. McWilliam, \& M. Rauch, 154

Oliveira, C. M., Dupuis, J., Chayer, P., \& Moos, H. W. 2005, ApJ, 625, 232

Padgett, D. L. 1996, ApJ, 471, 847

Paulson, D. B., Saar, S. H., Cochran, W. D., \& Hatzes, A. P. 2002, AJ, 124, 572

Pont, F., \& Eyer, L. 2004, MNRAS, 351, 487 
Rhode, K. L., Herbst, W., \& Mathieu, R. D. 2001, AJ, 122, 3258

Rich, R. M., \& Origlia, L. 2005, ApJ, 634, 1293

Roy, J.-R., \& Kunth, D. 1995, A\&A, 294, 432

Saar, S. H., \& Donahue, R. A. 1997, ApJ, 485, 319

Santos, N. C., Mayor, M., Naef, D., et al. 2000, A\&A, 361, 265

Santos, N. C., Israelian, G., \& Mayor, M. 2001, A\&A, 373, 1019

Santos, N. C., Israelian, G., \& Mayor, M. 2004, A\&A, 415, 1153

Santos, N. C., Israelian, G., Mayor, M., et al. 2005, A\&A, 437, 1127

Santos, N. C., Ecuvillon, A., Israelian, G., et al. 2006, A\&A, 458, 997

Setiawan, J., Henning, T., Launhardt, R., et al. 2008, Nature, 451, 38

Sicilia-Aguilar, A., Hartmann, L. W., Szentgyorgyi, A. H., et al. 2005, AJ, 129, 363

Sneden, C. 1973, Ph.D. Thesis, Univ. of Texas
Sofia, U. J., \& Meyer, D. M. 2001, ApJ, 554, L221

Sousa, S. G., Santos, N. C., Israelian, G., Mayor, M., \& Monteiro, M. J. P. F. G. 2006, A\&A, 458, 873

Steenbock, W., \& Holweger, H. 1981, A\&A, 99, 192

Torres, C. A. O., Quast, G. R., da Silva, L., et al. 2006, A\&A, 460, 695

Twarog, B. A., Ashman, K. M., \& Anthony-Twarog, B. J. 1997, AJ, 114, 2556

Udry, S., \& Santos, N. 2007, ARA\&A, 45, 397

Udry, S., Mayor, M., Naef, D., et al. 2000, A\&A, 356, 590

Udry, S., Mayor, M., \& Santos, N. 2003, A\&A, 407, 369

Wielen, R., Fuchs, B., \& Dettbarn, C. 1996, A\&A, 314, 438

Zoccali, M., Lecureur, A., Barbuy, B., et al. 2006, A\&A, 457, L1

Zuckerman, B., \& Song, I. 2004, ARA\&A, 42, 685 